Research Paper

\title{
Glucose-6-Phosphate Dehydrogenase Deficiency and Diabetes Mellitus with Severe Retinal Complications in a Sardinian Population, Italy
}

\author{
Antonio Pinna ${ }^{1 凶}$, Emma Luigia Contini ${ }^{1}$, Ciriaco Carru ${ }^{2}$, Giuliana Solinas ${ }^{3}$ \\ 1. Department of Surgical, Microsurgical, \& Medical Sciences, Section of Ophthalmology, University of Sassari, Sassari, Italy \\ 2. Department of Biomedical Sciences, Section of Clinical Biochemistry, University of Sassari, Sassari, Italy. \\ 3. Department of Biomedical Sciences, Laboratory of Epidemiology and Biostatistics, University of Sassari, Sassari, Italy
}

$\triangle$ Corresponding author: Antonio Pinna, MD, Institute of Ophthalmology, University of Sassari, Viale San Pietro 43 A, 07100 Sassari, Italy. Phone: 0039 079228251. Fax: 0039 079228484. E-mail: apinna@uniss.it

() Ivyspring International Publisher. This is an open-access article distributed under the terms of the Creative Commons License (http://creativecommons.org/ licenses/by-nc-nd/3.0/). Reproduction is permitted for personal, noncommercial use, provided that the article is in whole, unmodified, and properly cited.

Received: 2013.05.28; Accepted: 2013.08.14; Published: 2013.11.27

\begin{abstract}
Background: Glucose-6-Phosphate Dehydrogenase (G6PD) deficiency is one of the most common human genetic abnormalities, with a high prevalence in Sardinia, Italy. Evidence indicates that G6PD-deficient patients are protected against vascular disease. Little is known about the relationship between G6PD deficiency and diabetes mellitus. The purpose of this study was to compare G6PD deficiency prevalence in Sardinian diabetic men with severe retinal vascular complications and in age-matched non-diabetic controls and ascertain whether G6PD deficiency may offer protection against this vascular disorder.
\end{abstract}

Methods: Erythrocyte G6PD activity was determined using a quantitative assay in 390 diabetic men with proliferative diabetic retinopathy (PDR) and 390 male non-diabetic controls, both aged $\geq 50$ years. Conditional logistic regression models were used to investigate the association between G6PD deficiency and diabetes with severe retinal complications.

Results: G6PD deficiency was found in 21 (5.4 \%) diabetic patients and $33(8.5 \%)$ controls $(P=0.09)$. In a univariate conditional logistic regression model, G6PD deficiency showed a trend for protection against diabetes with PDR, but the odds ratio $(O R)$ fell short of statistical significance $(\mathrm{OR}=0.6,95 \%$ confidence interval $=0.35-1.08, P=0.09)$. In multivariate conditional logistic regression models, including as covariates G6PD deficiency, plasma glucose, and systemic hypertension or systolic or diastolic blood pressure, G6PD deficiency showed no statistically significant protection against diabetes with PDR.

Conclusions: The prevalence of G6PD deficiency in diabetic men with PDR was lower than in age-matched non-diabetic controls. G6PD deficiency showed a trend for protection against diabetes with PDR, but results were not statistically significant.

Key words: Glucose-6-Phosphate Dehydrogenase (G6PD) deficiency; diabetes mellitus; proliferative diabetic retinopathy; conditional logistic regression analysis

\section{Introduction}

Glucose-6-Phosphate Dehydrogenase (G6PD) is a cytoplasmatic enzyme affecting the production of the reduced form of the extramitochondrial Nicotine-Adenosine-Dinucleotide Phosphate coenzyme
(NADPH) by controlling the step from glucose-6-phosphate to 6-phosphogluconate in the pentose phosphate pathway. In erythrocytes, defense against oxidative damage is heavily dependent on 
G6PD activity, which is the only source of NAPDH. ${ }^{1}$ The gene encoding G6PD is located in the telomeric region of the long arm of the $\mathrm{X}$ chromosome (band Xq28). More than 300 alleles with point mutation in the G6PD gene sequence have been identified. ${ }^{2}$ The Gd-Mediterranean allele, associated with levels of enzyme activity undetectable with routine methods, is common among the male population in Sardinia, Italy, where the reported prevalence of G6PD deficiency is $8-15 \% .^{3-6}$ Hemizygous males have populations of uniformly deficient erythrocytes; conversely, heterozygous females have mosaic populations of normal and G6PD-deficient erythrocytes, due to random $X$ chromosome inactivation. G6PD deficiency is a public health issue in Sardinia, because of the seasonal occurrence of hemolytic crises after the ingestion of broad beans (Vicia faba) in G6PD-deficient subjects. Other important clinical manifestations of G6PD deficiency include neonatal jaundice and drug-induced hemolysis, which may follow the ingestion of agents with oxidant properties, such as primaquine, sulphonamides, nitrofurantoin, and several anti-inflammatory agents. ${ }^{2}$ The geographic distribution of G6PD deficiency, which is very similar to the distribution of current or past malaria endemicity, suggests that G6PD deficiency confers resistance to infection with falciparum malaria. ${ }^{2}$

Diabetes mellitus is the most common endocrine disorder in industrialized countries. The definition of diabetes mellitus has recently changed considerably. ${ }^{7}$ This condition is now defined as a group of metabolic diseases characterized by hyperglycemia resulting from defects in insulin secretion and/or action. Two main forms are recognized. Type 1 diabetes, previously called insulin-dependent diabetes mellitus or juvenile-onset diabetes, is due to a deficiency in endogenous insulin secretion secondary to destruction of insulin-producing beta cells in the pancreas. Although type 1 diabetes does have a peak incidence around the time of puberty, approximately $25 \%$ of cases present after 35 years of age. Type 2 diabetes, formerly known as non-insulin-dependent or adult-onset diabetes mellitus, is characterized by insulin resistance with an insulin secretory defect leading to relative insulin deficiency. This group accounts for $90-95 \%$ of patients with diabetes and also has a strong genetic predisposition. Type 2 patients are usually, but not always, older than age 40 at presentation. Obesity is a frequent finding and, in the United States, is present in $80-90 \%$ of these patients.

The relationship between G6PD deficiency and diabetes is still matter of debate. The hypothesis that hyperglycemia can lead to a decrease of G6PD activity is supported by experimental observations. ${ }^{8}$ The reverse hypothesis - that G6PD deficiency could be a risk factor for the occurrence of diabetes - has also been raised. In several populations, systematic screening of G6PD activity suggested an increased prevalence of G6PD deficiency in individuals with diabetes, compared with the background rate of the general population. ${ }^{8}$

Diabetic retinopathy is the leading cause of new cases of legal blindness among working-age people in developed countries. Proliferative diabetic retinopathy (PDR), the most vision-threatening form of diabetic retinopathy, has been reported to be present in approximately $50 \%$ of type 1 patients with 25 years' duration of the disease and in $25 \%$ of patients who have type 2 diabetes mellitus for 25 years or more. 9,10

Evidence indicates that patients with G6PD deficiency are protected against ischemic heart and cerebrovascular disease, retinal vein occlusion (RVO), and nonarteritic anterior ischemic optic neuropathy (NAION). ${ }^{4,11-13}$ On the other hand, an increased prevalence of PDR in G6PD-deficient patients with type 1 diabetes has recently been reported in a small study, suggesting that G6PD deficiency accelerates the retinal microvascular complications of diabetes. ${ }^{14}$

The purpose of this study was to compare G6PD deficiency prevalence in Sardinian diabetic men with severe retinal vascular complications and in age-matched non-diabetic controls and ascertain whether G6PD deficiency may offer protection against this vascular disorder.

\section{Methods}

The present study used a case-control design, recruiting 390 out of 420 consecutive diabetic men with severe retinal complications and 390 male age-matched non-diabetic controls between January 1994 and December 2008. Both patients and controls were aged $\geq 50$ years and of Sardinian ancestry. Women were excluded because of the small number of homozygote subjects with complete lack of erythrocyte G6PD activity. Sample size was computed before the survey with a 95\% confidence level (two-tailed test) and 82\% statistical power to detect an odds ratio of 2, assuming a G6PD prevalence rate of $8.5 \%$, as reported previously. ${ }^{3-6}$ The case-control ratio was 1:1.

The inclusion criteria for the case group were diagnosis of type 1 or type 2 diabetes mellitus with PDR, full Sardinian ancestry, and age $\geq 50$ years. According to the Early Treatment of Diabetic Retinopathy Study (ETDRS) classification, the diagnosis of PDR was established by the detection of new vessels at the optic disk and/or elsewhere in the retina on ophthalmoscopic examination and fluorescein angiography. ${ }^{15,16}$ Plasma glucose, glycated hemoglobin $\left(\mathrm{HbA}_{1 \mathrm{c}}\right)$, systolic and diastolic blood pressure, and 
medical conditions, including systemic hypertension, hypercholesterolemia, and cardio- and cerebrovascular status were recorded. All diabetic patients underwent a full ophthalmic evaluation, including best corrected visual acuity (BCVA), slit-lamp examination, applanation tonometry, fundus biomicroscopy, and fluorescein angiography. Exclusion criteria included age $<50$ years, any level of non-Sardinian ancestry, and evidence of any other retinal vascular disorder.

Age-matched controls were randomly selected from patients undergoing cataract surgery. Exclusion criteria included clinical/laboratory evidence of diabetes mellitus, age $<50$ years, any level of non-Sardinian ancestry, and previous history of retinal artery occlusion, RVO, or anterior ischemic optic neuropathy. All controls underwent standard ophthalmic evaluation, including BCVA, slit-lamp examination, applanation tonometry, and fundus examination. Plasma glucose, systolic and diastolic blood pressure, and medical conditions were also recorded. Controls were recruited concurrently during the patients' recruitment period.

Subjects were classified as diabetic if they were under treatment for type 1 or type 2 diabetes or if they had a fasting plasma glucose level of $\geq 126 \mathrm{mg} / \mathrm{dL}$ and/or a plasma glucose level of $\geq 200 \mathrm{mg} / \mathrm{dL} 2$ hours after a 75-g oral glucose load in a glucose tolerance test (as defined by the WHO). Subjects were considered to have hypertension if they were receiving treatment with anti-hypertension drugs or if their blood pressure was $>140 \mathrm{~mm} \mathrm{Hg}$ systolic or $>90 \mathrm{~mm}$ $\mathrm{Hg}$ diastolic (as defined by the $\mathrm{WHO} /$ International Society of Hypertension). Hypercholesterolemia was defined by a fasting plasma cholesterol level of $\geq 220$ $\mathrm{mg} / \mathrm{dL}$ or the intake of lipid-lowering drugs.

Institutional ethics review board approval was obtained and the study was conducted in full accord with the tenets of the Declaration of Helsinki. Each participant received detailed information and provided informed consent before inclusion.

Seven percent of the cases and $5 \%$ of the controls who were eligible for the study declined to participate. The major reason was "not interested."

Red blood cell G6PD activity was determined using a quantitative assay (G6PD/6PGD, Biomedic snc, Sassari, Italy) as described previously.6,11,12 Quantitative testing for G6PD deficiency is routinely performed in all patients admitted to our hospital.

Categorical values were compared by Chi-square test. The differences between cases and controls for quantitative variables were analyzed by Student's $t$ test. Univariate conditional logistic regression models were first used to investigate the association between diabetes with PDR and some vari- ables, including G6PD deficiency, plasma glucose, systolic or diastolic blood pressure and systemic hypertension. Multivariate conditional logistic regression analysis, including as covariates plasma glucose and systemic hypertension or systolic or diastolic blood pressure, were used to determine the significance of the association between G6PD deficiency and diabetes with PDR. ${ }^{17}$ Odds ratios (ORs) and 95\% confidence intervals (CIs) were calculated. $P$ values $\leq 0.05$ were considered to be statistically significant. Statistical analysis was performed with commercial software (STATA ver. 9.0; StataCorp, College Station, TX).

\section{Results}

The study group consisted of 390 patients (mean age: $63.6 \pm 7.3$ years), all with bilateral PDR; 58 (14.9\%) had type 1 diabetes and 332 (85.1\%) had type 2 diabetes. Diabetes duration was $\geq 15$ years in all patients. Mean visual acuity was $0.42 \pm 0.35$ (range: $0-1$ ) in the right eye and $0.41 \pm 0.35$ (range: $0-1$ ) in the left eye. The right and left eyes had almost identical mean intraocular pressure values $(15.7 \pm 5.3 \mathrm{~mm} \mathrm{Hg}$ and $15.2 \pm 4.1$ $\mathrm{mm} \mathrm{Hg}$ ). Neovascular glaucoma was found in 17 $(4.4 \%)$ patients; in two, this condition was bilateral. Two patients were aphakic and 103 (26.4\%) had a posterior chamber intraocular lens; of these, 57 had bilateral pseudophakia. Vitreous hemorrhage was observed in $96(24.6 \%)$ patients, of whom 30 had bilateral involvement. Overall, vitreous hemorrhage was seen in 67 right eyes and 59 left ones.

The patients' and controls' systemic characteristics are reported in Table 1. Diabetic patients had a significantly higher frequency of systemic hypertension and significantly higher levels of systolic and diastolic blood pressure and plasma glucose than the control subjects. Hypercholesterolemia was disclosed in $27.9 \%$ of patients with PDR. Unfortunately, data about hypercholesterolemia in the control population were incomplete; consequently, it was not possible to include this risk factor in the statistical analysis. Mean $\mathrm{HbA}_{1 \mathrm{c}}$ in diabetic patients with PDR was $7.9 \pm 1.1 \%$, a result indicating poor glycemic control.

G6PD deficiency was found in 21 (5.4\%) out of 390 diabetic patients; of these, $3(5.2 \%)$ had type 1 diabetes and $18(5.4 \%)$ had type 2 diabetes. On the other hand, G6PD deficiency was found in 33 (8.5\%) out of 390 controls, a prevalence rate within the range observed in the Sardinian male population..$^{3-6}$ All G6PD-deficient men had undetectable levels of red blood cell enzyme activity (total deficiency). The data indicated a higher prevalence of G6PD deficiency in controls compared to diabetic patients with PDR, but it was not significant at the 0.05 significance level $(P=$ 0.09). None of the patients with G6PD deficiency had shown clinical manifestations of favism or 
drug-induced hemolysis in the two years before their enrolment in this study.

In a univariate conditional logistic regression model, G6PD deficiency showed a trend for protection against diabetes with PDR, but the OR was not statistically significant $(\mathrm{OR}=0.62,95 \% \mathrm{CI}=0.35-1.08$, $P=0.09$ ).

Multivariate conditional logistic regression results are reported in Tables 2-4. In these models, including as covariates G6PD deficiency, plasma glucose levels, and systemic hypertension or systolic or diastolic blood pressure values, G6PD deficiency showed a trend for protection against diabetes with PDR, but the ORs were not statistically significant. Conversely, elevated plasma glucose, systemic hypertension, and increased diastolic blood pressure were significantly associated with increased risk for diabetes with PDR.

Table I. Systemic characteristics of diabetic patients with proliferative diabetic retinopathy (PDR) and control subjects.

\begin{tabular}{|c|c|c|c|}
\hline & $\begin{array}{l}\text { Diabetic } \\
\text { patients } \\
(n=390)\end{array}$ & $\begin{array}{l}\text { Controls } \\
(\mathrm{n}=390)\end{array}$ & $\begin{array}{l}P \\
\text { Cases vs. } \\
\text { Controls }\end{array}$ \\
\hline Age, years (mean) $\pm S D$ & $63.6 \pm 7.3$ & $64.1 \pm 6.9$ & 0.28 \\
\hline $\begin{array}{l}\text { Plasma glucose, } \mathrm{mg} / \mathrm{dL} \text { (mean) } \\
\pm \mathrm{SD}\end{array}$ & $157.8 \pm 3.6$ & $98.3 \pm 0.7$ & $<0.0001$ \\
\hline $\begin{array}{l}\text { Systolic blood pressure, } \mathrm{mm} \\
\mathrm{Hg} \text { (mean) } \pm \text { SD }\end{array}$ & $139.9 \pm 1.0$ & $133.7 \pm 0.9$ & $<0.0001$ \\
\hline $\begin{array}{l}\text { Diastolic blood pressure, } \mathrm{mm} \\
\mathrm{Hg} \text { (mean) } \pm \mathrm{SD}\end{array}$ & $81.3 \pm 0.5$ & $78.3 \pm 0.5$ & $<0.0001$ \\
\hline G6PD deficiency, n (\%) & $21(5.4)$ & $33(8.5)$ & 0.09 \\
\hline Systemic hypertension, $\mathbf{n}(\%)$ & $313(80.3)$ & $150(38.5)$ & $<0.001$ \\
\hline Hypercholesterolemia, n (\%) & $109(27.9)$ & -- & --- \\
\hline $\mathrm{HbA}_{1 \mathrm{c}}(\%)$ & $7.9 \pm 1.1$ & --- & --- \\
\hline
\end{tabular}

Table 2. Multivariate conditional logistic regression analysis (including G6PD deficiency, systemic hypertension, and plasma glucose), showing odds ratios for diabetes with proliferative diabetic retinopathy (PDR; $n=390)$. Number of controls: 390 .

\begin{tabular}{llll}
\hline & Odds Ratio & $\begin{array}{l}\text { PDR } \\
\text { 95\% Confidence } \\
\text { Interval }\end{array}$ & P value \\
\hline $\begin{array}{l}\text { G6PD deficiency } \\
\text { (yes/no) }\end{array}$ & 0.63 & $0.26-1.57$ & 0.32 \\
$\begin{array}{l}\text { Systemic hypertension } \\
\text { (yes/no) }\end{array}$ & 6.3 & $3.75-10.62$ & $<0.0001$ \\
$\begin{array}{l}\text { Plasma glucose } \\
\text { (mg/dl) }\end{array}$ & 1.03 & $1.03-1.04$ & $<0.0001$ \\
\hline
\end{tabular}

Table 3. Multivariate conditional logistic regression analysis (including G6PD deficiency, systolic blood pressure, and plasma glucose), showing odds ratios for diabetes with proliferative diabetic retinopathy (PDR; $n=390)$. Number of controls: 390 .

\begin{tabular}{|c|c|c|c|}
\hline & & PDR & \\
\hline Factor & Odds Ratio & $\begin{array}{l}\text { 95\% Confidence } \\
\text { Interval }\end{array}$ & P value \\
\hline $\begin{array}{l}\text { G6PD deficiency } \\
\text { (yes/no) }\end{array}$ & 0.85 & $0.39-1.84$ & 0.68 \\
\hline $\begin{array}{l}\text { Systolic blood } \\
\text { pressure }(\mathrm{mm} \mathrm{Hg})\end{array}$ & 1.01 & 0.99-1.02 & 0.11 \\
\hline $\begin{array}{l}\text { Plasma glucose } \\
(\mathrm{mg} / \mathrm{dl})\end{array}$ & 1.03 & $1.03-1.04$ & $<0.0001$ \\
\hline
\end{tabular}

Table 4. Multivariate conditional logistic regression analysis (including G6PD deficiency, diastolic blood pressure, and plasma glucose), showing odds ratios for diabetes with proliferative diabetic retinopathy (PDR; $\mathrm{n}=390)$. Number of controls: 390 .

\begin{tabular}{llll}
\hline & Odds Ratio & $\begin{array}{l}\text { PDR } \\
\text { 95\% Confidence } \\
\text { Interval }\end{array}$ & P value \\
\hline $\begin{array}{l}\text { G6PD deficiency } \\
\text { (yes/no) }\end{array}$ & 0.87 & $0.40-1.88$ & 0.72 \\
$\begin{array}{l}\text { Diastolic blood } \\
\text { pressure (mm Hg) }\end{array}$ & 1.02 & $0.99-1.04$ & 0.05 \\
$\begin{array}{l}\text { Plasma glucose } \\
\text { (mg/dl) }\end{array}$ & 1.03 & $1.03-1.04$ & $<0.0001$ \\
\hline
\end{tabular}

\section{Discussion}

The most common enzyme deficiency in humans, G6PD deficiency affects an estimated 400 million people worldwide. ${ }^{2}$ This disorder is found mainly in the tropical and sub-tropical regions of the world, with the highest rates, usually 5-30\%, in Africa, Asia, the Middle East, the Mediterranean, and Papua New Guinea. ${ }^{18,19}$ In the U.S., black males are commonly affected, with a prevalence rate of $10 \% .{ }^{19}$ Sardinia is one of the areas with the highest prevalence, with rates ranging from $8 \%$ to $15 \%$. $^{3-6}$ Former studies have suggested that the geographic distribution of G6PD deficiency, which is highly correlated with the distribution of current or past malaria endemicity, is the result of a balanced polymorphism conferring resistance to infection with falciparum malaria. ${ }^{2}$

A frequent cause of blindness in the U.S., diabetic retinopathy is the leading cause in patients aged 20-64 years. The exact pathogenic mechanism of diabetic microvascular disease is unknown. Exposure to hyperglycemia over an extended period is believed to result in biochemical and physiological changes that ultimately cause endothelial damage. Specific retinal capillary changes include basement membrane thickening and selective loss of pericytes, which favor 
capillary occlusion and retinal nonperfusion, as well as decompensation of the endothelial barrier function, which allows serum leakage and retinal edema to occur. A large number of hematologic and biochemical abnormalities have been associated with the prevalence and severity of diabetic retinopathy, such as increased erythrocyte aggregation, increased platelet adhesiveness, defective fibrinolysis, abnormal serum lipids, abnormalities in serum and whole blood viscosity, abnormal levels of growth hormone, and upregulation of vascular endothelial growth factor (VEGF). ${ }^{20}$

There is general agreement that the severity of hyperglycemia is the key alterable risk factor associated with the development and progression of diabetic retinopathy. ${ }^{21-23}$ Furthermore, intensive management of systemic hypertension has been demonstrated to slow diabetic retinopathy progression. ${ }^{24,25}$ In our study, diabetic patients with PDR had a significantly higher frequency of systemic hypertension and significantly higher values of systolic and diastolic blood pressure and plasma glucose than the control subjects. Likewise, multivariate conditional logistic regression analysis showed that hyperglycemia, systemic hypertension, and elevated diastolic blood pressure were significantly associated with an increased risk of diabetes with severe retinal vascular complications. Our results are consistent with earlier studies, confirming that elevated levels of plasma glucose and systemic hypertension, especially diastolic hypertension, are important risk factors for PDR. ${ }^{21-24}$

The role of G6PD deficiency in the pathogenesis of diabetic vascular disease is far from clear. Former research showed no association between diabetes mellitus and G6PD deficiency. ${ }^{26}$ Theoretically, this genetic abnormality should represent a disadvantage, since NADPH is necessary to regenerate reduced glutathione and decreased NADPH production may facilitate oxidative stress. ${ }^{1}$ Furthermore, G6PD deficiency might worsen diabetic vascular disease by increasing endothelial dysfunction, as the decreased supply of G6PD-derived NADPH, a cofactor for endothelial nitric oxide $(\mathrm{NO})$ synthase, may reduce the synthesis of NO, a potent vasodilator with antiatherogenic effects. ${ }^{27,28}$ On the other hand, experimental and clinical evidence indicates that G6PD deficiency may attenuate the process leading to diabetic microangiopathy. Indeed, the decreased supply of NADPH may damp the activity of aldose reductase in the first step of the polyol pathway, thus limiting the contribution of the excess of polyols to the pathogenesis of diabetic vascular damage. ${ }^{29}$ Furthermore, the decreased production of NADPH might paradoxically protect against oxidative stress, as suggested by a recent study associating elevated G6PD activity and elevated NDAPH levels with endothelial and vascular dysfunction in diabetic patients. ${ }^{30}$ Indeed, G6PD-derived NADPH, a cofactor for NADPH oxidase, enhances superoxide anion generation and elevates oxidative stress. ${ }^{30}$ G6PD deficiency might also offer protection against diabetic microangiopathy because of the decreased cholesterol synthesis. ${ }^{5}$

In a recently published paper, Cappai et al. ${ }^{14}$ investigated the prevalence of PDR in patients with type 1 diabetes of duration $\geq 15$ years, who were G6PD-deficient $(n=19)$ or -sufficient $(n=35)$. They found an increased prevalence of PDR in G6PD-deficient patients, suggesting that G6PD deficiency is a risk factor for PDR, because it accelerates the retinal microvascular complications of diabetes.

In our large study, we found that the prevalence of G6PD deficiency in diabetic men with PDR was lower than expected. Indeed, there was a higher prevalence of G6PD deficiency in non-diabetic controls, compared to diabetics with PDR. In a univariate conditional logistic regression model, G6PD deficiency showed a trend for protection against diabetes with PDR $(P=0.09)$. However, in multivariate conditional logistic regression models, including as covariates G6PD deficiency, plasma glucose levels, and systemic hypertension or systolic or diastolic blood pressure, results showed that G6PD deficiency does not confer any significant protection against diabetes with PDR. Overall, if it is true that our results failed to demonstrate that G6PD deficiency may offer protection against diabetes with PDR, it is also true that we found no evidence that it may be a risk factor for this condition.

Recent studies have suggested that G6PD-deficient patients are protected against ischemic heart and cerebrovascular disease, RVO, and NAION. ${ }^{4,11-13}$ Unlike in RVO and NAION, G6PD deficiency seems not to confer protection against the severe retinal vascular complications of diabetes. This discrepancy might be explained by the different pathogenic mechanisms underlying these different retinal vascular disorders. On the one hand, in PDR, retinal endothelial damage is largely due to long-lasting hyperglycemia; ${ }^{20}$ on the other hand, in RVO and NAION, atherosclerosis is believed to play a key role in the pathogenesis of the vascular damage. ${ }^{11,12}$ Actually, in former studies, we found hypercholesterolemia in $35.7 \%$ and $34.3 \%$ of patients with RVO and NAION, respectively; ${ }^{11,12}$ conversely, in the present survey, hypercholesterolemia was disclosed in only $27.9 \%$ of diabetic patients with PDR.

Hypercholesterolemia, a well-known risk factor for cardiovascular disease, damages small blood vessels by impairing endothelial vasodilator function, 
probably by interfering with the synthesis of $\mathrm{NO} .{ }^{28} \mathrm{~A}$ large body of experimental evidence linking G6PD activity, cholesterol synthesis, and cell growth has accumulated in recent years. ${ }^{5,31}$ Interestingly, Batetta et al. ${ }^{5}$ have shown that the Mediterranean variant of G6PD deficiency is characterized by peculiar alterations in plasma and intracellular cholesterol metabolism, such as reduced synthesis and esterification. In G6PD-deficient subjects, the reduced ability to esterify and accumulate cholesterol in the arteries may account for a lower risk of atherosclerotic disease and, therefore, RVO and NAION.11,12 Overall, it is likely that in diabetic G6PD-deficient men with PDR the retinal endothelial damage caused by long-standing hyperglycemia outweighs the protection offered by G6PD deficiency against retinal vascular disorders.

As in other studies analyzing the relationship between retinal vascular disorders and certain vascular risk factors, the control group was selected from patients undergoing cataract surgery. ${ }^{11,12,32-34}$ It is highly unlikely that this strategy may have introduced a selection bias, because the prevalence rate $(8.5 \%)$ of G6PD deficiency in the control group was within the range observed in the Sardinian male population. ${ }^{3-6}$ This finding rules out the hypothesis that G6PD deficiency might result in increased susceptibility to cataract, in full agreement with former studies clearly showing that Sardinian G6PD-deficient patients do not have a higher risk of developing cataract. 6,35

One could argue that we compared the prevalence of G6PD deficiency in diabetes (with the qualifier "and retinal vascular complications") versus non-diabetes, rather than G6PD deficiency prevalence in diabetic patients with PDR versus diabetic patients who have not developed PDR after the same duration of diabetes. However, the latter comparison would apply only if the prevalence of G6PD deficiency was not altered in diabetes, which still remains to be established. Keeping this fact into account, we strongly believe that the correct comparison is to be made between diabetic and non-diabetic subjects, and this is what we performed in our investigation.

Our study has several important limitations. First of all, it was restricted to a limited, genetically homogeneous group of male patients (i.e. those of Sardinian ancestry); as a result, our findings may not be applicable to diabetic patients of non-Sardinian ancestry. Furthermore, we analyzed a sample of subjects aged 50 years or older; however, the exclusion of individuals under the age of 50 may be considered of little importance for the purpose of our study, as G6PD deficiency does not influence the life expectancy of the affected subjects and its frequency does not increase with age. 6 Moreover, in our statistical models, type 1 and type 2 diabetes cases were pooled together, because the two subgroups showed almost identical G6PD deficiency prevalence rates. This approach may be questionable, because type 1 and type 2 diabetes are two different clinical entities with different pathogenesis, natural history, and PDR incidence. Another potential limitation is the fact that we did not assess the impact of tobacco smoking. Last, but not least, data about hypercholesterolemia in the control population were incomplete; as a result, it was not possible to include this risk factor in the logistic regression models.

In conclusion, we found that the prevalence of G6PD deficiency in Sardinian diabetic men with PDR was lower than in age-matched non-diabetic controls. G6PD deficiency showed a trend for protection against diabetes with PDR, but results were not statistically significant. Our data also support earlier reports associating hyperglycemia and systemic hypertension with the severe retinal complications of diabetes, ${ }^{21-25}$ but contrast with other studies suggesting that G6PD deficiency is a risk factor for PDR. ${ }^{14}$ Further experimental and clinical studies are necessary for a better understanding of the mechanism by which G6PD deficiency may affect diabetes and its retinal vascular complications.

\section{Acknowledgements}

This study was in part supported by grant CRP-25871 funded by Regione Autonoma della Sardegna, Italy

\section{Competing Interests}

The authors have declared that no competing interest exists.

\section{References}

1. Kletzien RF, Harris PK, Foellmi LA. Glucose-6-phosphate dehydrogenase: a "housekeeping" enzyme subject to tissue-specific regulation by hormones, nutrients, and oxidant stress. FASEB J. 1994; 8: 174-81.

2. Beutler E. G6PD deficiency. Blood. 1994; 84: 3613-36.

3. Maida A, Pettinato S, Bo G. Clinical manifestations of favism and G6PD deficiency: epidemiological survey in the province of Sassari (Sardinia, Italy). Haematologica. 1973; 58: 1265-82.

4. Cocco P, Todde P, Fornera S, Manca MB, Manca M, Sias AR. Mortality in a cohort of men expressing the Glucose-6-Phosphate Dehydrogenase deficiency. Blood. 1998; 91: 706-9.

5. Batetta B, Bonatesta RR, Sanna F, Putzolu M, Mulas MF, Collu M, Dessì S. Cell growth and cholesterol metabolism in human glucose-6-phosphate dehydrogenase deficient lymphomononuclear cells. Cell Prolif. 2002; 35: 143-54.

6. Pinna A, Pes A, Zinellu A, Carta A, Solinas G. Glucose-6-Phosphate Dehydrogenase (G6PD) deficiency and senile cataract in a Sardinian male population, Italy. Ophthalmic Epidemiol. 2009; 16: 395-9.

7. Expert Committee on the Diagnosis and Classification of Diabetes Mellitus. Report of the Expert Committee on the Diagnosis and Classification of Diabetes Mellitus. Diabetes Care. 2003; 26 (Suppl 1):S5-S20.

8. Carette C, Dubois-Laforgue D, Gautier JF, Timsit J. Diabetes mellitus and glucose-6-phosphate dehydrogenase deficiency: from one crisis to another. Diabetes Metab. 2011; 37: 79-82.

9. Klein R, Klein BE, Moss SE, Davis MD, DeMets DL. The Wisconsin epidemiologic study of diabetic retinopathy. II. Prevalence and risk of diabetic retinopathy when age at diagnosis is less than 30 years. Arch Ophthalmol. 1984; 102: 520-6.

10. Klein R, Klein BE, Moss SE, Davis MD, DeMets DL. The Wisconsin epidemiologic study of diabetic retinopathy. III. Prevalence and risk of diabetic reti- 
nopathy when age at diagnosis is 30 or more years. Arch Ophthalmol. 1984; 102: 527-32.

11. Pinna A, Carru C, Solinas G, Zinellu A, Carta F. Glucose-6-phosphate dehydrogenase deficiency in retinal vein occlusion. Invest Ophthalmol Vis Sci. 2007; 48: 2747-52.

12. Pinna A, Solinas G, Masia C, Zinellu A, Carru C, Carta A. Glucose-6-phosphate dehydrogenase (G6PD) deficiency in nonarteritic anterior ischemic optic neuropathy in a Sardinian population, Italy. Invest Ophthalmol Vis Sci. 2008; 49: 1328-32.

13. Meloni L, Manca MR, Loddo I, Cioglia G, Cocco P, Schwartz A, Muntoni S, Muntoni S. Glucose-6-phosphate dehydrogenase deficiency protects against coronary heart disease. J Inherit Metab Dis. 2008; 31: 412-7.

14. Cappai G, Songini M, Doria A, Cavallerano JD, Lorenzi M. Increased prevalence of proliferative retinopathy in patients with type 1 diabetes who are deficient in glucose-6-phosphate dehydrogenase. Diabetologia. 2011; 54: 1539-42.

15. Early Treatment Diabetic Retinopathy Study Research Group. Early Treatment Diabetic Retinopathy Study design and baseline patient characteristics. ETDRS report number 7. Ophthalmology. 1991; 98(5 Suppl): 741-56.

16. Early Treatment Diabetic Retinopathy Study Research Group. Grading diabetic retinopathy from stereoscopic color fundus photographs - an extension of the modified Airlie House classification. ETDRS report number 10. Ophthalmology. 1991; 98(5 Suppl): 786-806.

17. Clayton D, Hills M. Statistical Models in Epidemiology. Oxford, UK: Oxford University Press; 1993

18. WHO Working Group. Glucose-6-phosphate dehydrogenase deficiency. Bull World Health Organ. 1989; 67: 601-11.

19. Ruwende C, Hill A. Glucose-6-phosphate dehydrogenase deficiency and malaria. J Mol Med. 1998; 76: 581-8.

20. Abu El-Asrar AM, Al-Mezaine HS, Ola MS. Pathophysiology and management of diabetic retinopathy. Exper Rev Ophthalmol. 2009; 4: 627-47.

21. Diabetes Control and Complications Trial/Epidemiology of Diabetes Interventions and Complications Research Group. Retinopathy and nephropathy in patients with type 1 diabetes four years after a trial of intensive therapy. $\mathrm{N}$ Engl J Med. 2000; 342: 381-9.

22. Diabetes Control and Complications Trial Research Groups. The relationship of glycemic exposure (HbA1c) to the risk of development and progression of retinopathy in the Diabetes Control and Complications Trial. Diabetes. 1995; 44: 968-83.

23. Wong TY, Liew G, Tapp RJ, Schmidt MI, Wang JJ, Mitchell P, Klein R, Klein $\mathrm{BE}$, Zimmet P, Shaw J. Relation between fasting glucose and retinopathy for diagnosis of diabetes: three population-based cross-sectional studies. Lancet. 2008; 371: 736-43.

24. UK Prospective Diabetes Study Group. Tight blood pressure control and risk of macrovascular and microvascular complications in type 2 diabetes: UKPDS 38. BMJ. 1998; 317: 703-13.

25. Snow V, Weiss KB, Mottur-Pilson C. The evidence for tight blood pressure control in the management of type 2 diabetes mellitus. Ann Int Med. 2003; 138 : 587-92

26. Meloni T, Pacifico A, Forteleoni G, Meloni GF. G6PD deficiency and diabetes mellitus in northern Sardinian subjects. Haematologica. 1992; 77: 94-5.

27. Leopold JA, Walker J, Scribner AW, Voetsch B, Zhang YY, Loscalzo AJ, Stanton RC, Loscalzo J. Glucose-6-phosphate dehydrogenase modulates vascular endothelial growth factor-mediated angiogenesis. J Biol Chem. 2003; 278: 32100-6.

28. Moncada $\mathrm{S}$, Higgs EA. The discovery of nitric oxide and its role in vascular biology. Br J Pharmacol. 2006; 147 (Suppl 1):S193-S201.

29. Kennedy A, Frank RN, Varma SD. Galactitol accumulation by glucose-6-phosphate deficient fibroblasts: a cellular model for resistance to the complications of diabetes mellitus. Life Sci. 1983; 33: 1277-83.

30. Gupte SA. Glucose-6-phosphate dehydrogenase: a novel therapeutic target in cardiovascular diseases. Curr Opin Investig Drugs. 2008; 9: 993-1000.

31. Rao KN. The significance of cholesterol biosynthesis pathway in cell growth and carcinogenesis (review). Anticancer Res. 1995; 15: 309-14.

32. The Eye Disease Case-Control Study Group. Risk factors for central retinal vein occlusion. Arch Ophthalmol. 1996; 114: 545-54.

33. Talks SJ, Chong NH, Gibson JM, Dodson PM. Fibrinogen, cholesterol and smoking as risk factors for nonarteritic anterior ischemic optic neuropathy. Eye. 1995; 9: 85-8.

34. Salomon O, Huna-Baron R, Kurtz S, Steinberg DM, Moisseiev J, Rosenberg N, Yassur I, Vidne O, Zivelin A, Gitel S, Davidson J, Ravid B, Seligsohn U. Analysis of prothrombotic and vascular risk factors in patients with nonarteritic ischemic optic neuropathy. Ophthalmology. 1999; 106: 739-42.

35. Meloni T, Carta F, Forteleoni G, Carta A, Ena F, Meloni GF. Glucose 6-phosphate dehydrogenase deficiency and cataract of patients in Northern Sardinia. Am J Ophthalmol. 1990; 110: 661-4. 